\title{
Micronization of sulfamethoxazole using the supercritical anti-solvent process
}

\author{
Yun-Ping Chang $\cdot$ Muoi Tang $\cdot$ Yan-Ping Chen
}

Received: 15 November 2006/Accepted: 13 August 2007/Published online: 22 December 2007

(C) Springer Science+Business Media, LLC 2007

\begin{abstract}
Micronization of an antibiotic compound sulfamethoxazole was investigated in this study using the supercritical anti-solvent (SAS) precipitation method. The results from either the batch or continuous process were compared, and the latter one yielded smaller particles. Effects on particle size due to various process parameters in the continuous SAS process (the concentration and the flow rate of the solution of sulfamethoxazole, the operating pressure and temperature) had been studied through a fractional factorial design for sulfamethoxazole. The experimental results showed that there was a significant interaction between the parameters of the flow rate and the concentration of the sulfamethoxazole solution. Analyses of the micronized sulfamethoxazole particles were examined using SEM, XRD and DSC. Sulfamethoxazole was micronized from its original size of 41.7 to $5.1 \mu \mathrm{m}$. The micronized sulfamethoxazole exhibited a higher dissolution rate in a simulated intestinal fluid than that of the original compound. It was further demonstrated that the coprecipitation of sulfamethoxazole with a hydrophilic polymer hydroxypropyl cellulose (HPC) in the continuous SAS process provided more enhanced dissolution rate.
\end{abstract}

\section{Introduction}

Supercritical fluid (SCF) technology is widely employed in materials processing where the formation of micronized particles plays an important role for specialty chemicals.

Y.-P. Chang - M. Tang · Y.-P. Chen $(\bowtie)$

Department of Chemical Engineering, National Taiwan

University, Taipei, Taiwan, ROC

e-mail: ypchen@ntu.edu.tw
The SCF technology has been applied in pharmaceutical processing $[1,2]$ to produce smaller particles with narrow size distribution. Its advantages over conventional technologies (mechanical comminution, spray drying, and solvent evaporation) include that trace residual solvent in the products and the simultaneous sterilization associated with the materials processing. The most commonly used SCF is carbon dioxide owing to its low critical properties and the safety consideration. When supercritical $\mathrm{CO}_{2}$ acts as the anti-solvent in the particle formation process, it is recognized as the supercritical anti-solvent (SAS) precipitation. Both the batch and continuous modes of the SAS process were applied in literature.

Pharmaceutical materials were micronized in the SAS process, together with changes in morphology and surface structures. These modifications influenced the bioavailabilities and dissolution rates of pharmaceuticals. In oral drug delivery, the dissolution rate of pharmaceuticals in the gastrointestinal tract is critical for absorption [3]. Improving the rate of dissolution in the biological environment can also reduce the required dose of oral drug. Particles with size distribution between 1 and $5 \mu \mathrm{m}$ are usually required for lung deliveries. This size range for pharmaceutical materials offers the possibility of avoiding the extensive gastrointestinal breakdown and high hepatic first-pass effect that dramatically limit the pharmacological activity of many drugs [4].

The SAS micronization processes have been successfully applied for various pharmaceutical compounds such as the non-steroidal anti-inflammatory drugs (NSAIDs), antibiotics, asthma drug, and protein [5]. The optimal conditions to produce smaller particles were essential to industrial process design. In this study, both the batch and continuous processes were employed for the micronization of sulfamethoxazole. Sulfamethoxazole is an effective antimicrobial agent used in treating a variety of bacterial 
infections. Micronization of this pharmaceutical material using the continuous SAS process was not observed in literature. The targets of this study are to compare the results between the batch and continuous operations, and to investigate the effects of process parameters on the final products using a fractional factorial experiment design approach. The dissolution rates of the micronized sulfamethoxazole particles were also measured and reported in a simulated intestinal fluid to justify its further application in pharmaceutical industry.

\section{Experimental section}

Materials

Sulfamethoxazole $\left(\mathrm{C}_{10} \mathrm{H}_{11} \mathrm{~N}_{3} \mathrm{O}_{3} \mathrm{~S}\right)$ was purchased from Sigma with the purity greater than 99 mass\%. Acetone (Merck, 99.8 mass\%) was used as the solvent in this study. Carbon dioxide with the purity better than 99.8 mass\% (San Fu, Taiwan) was used as the anti-solvent. Potassium phosphate monobasic (Merck, 99.5 mass\%) and sodium hydroxide (Merck, minimum 98 mass\%) were used in preparing the phosphate buffer solution for the measurement of the dissolution rate of sulfamethoxazole. Hydroxypropyl cellulose (Hercules, molecular weight of $10^{6}$ ) was used as additive in the co-precipitation study.

Apparatus and procedures

\section{Batch SAS process}

The batch type experimental apparatus had been presented in our previous study [6]. It consisted of three sections for carbon dioxide supply, precipitation, and depressurization. The precipitator was a stainless steel tube with volume of $75 \mathrm{~mL}$. The micronized particles were collected on the frits at the bottom of the tube. The frits had pore sizes of 0.1 and $0.5 \mu \mathrm{m}$.

In the batch SAS process, $5 \mathrm{~mL}$ solution of sulfamethoxazole in acetone was charged into the precipitator. Acetone was used in this study because sulfamethoxazole had larger solubility in this solvent. Carbon dioxide was then injected from the bottom of the precipitator where pressure increased from atmospheric condition to 120 bar within $20 \mathrm{~s}$. Volume expansion of the solution resulted in the recrystallization of sulfamethoxazole, and complete precipitation was expected after $30 \mathrm{~min}$. The micronized particles were then dried by flowing $\mathrm{CO}_{2}$ continuously through the top of the precipitator for $4 \mathrm{~h}$ before they were collected for analyses.

\section{Continuous SAS process}

The schematic diagram of our continuous type apparatus is shown in Fig. 1. A high flow rate HPLC pump (SSI, Prep 100) was used for $\mathrm{CO}_{2}$ delivery and another HPLC pump (SSI, series II) was used for pumping the solution of sulfamethoxazole. The precipitator was the same as that in the batch process. The $\mathrm{CO}_{2}$ flow rate was measured by a rotameter and was adjusted to $4 \mathrm{~L} / \mathrm{min}$ (measured at the ambient conditions) using the micrometering valve at the exit of the precipitator. Pressure in the precipitator was regulated by a back pressure valve (Tescom). The precipitator was immersed in a water bath. The accuracies in pressure and temperature were controlled at \pm 0.1 bar and $\pm 0.1 \mathrm{~K}$,
Fig. 1 Experimental apparatus of the continuous SAS processes

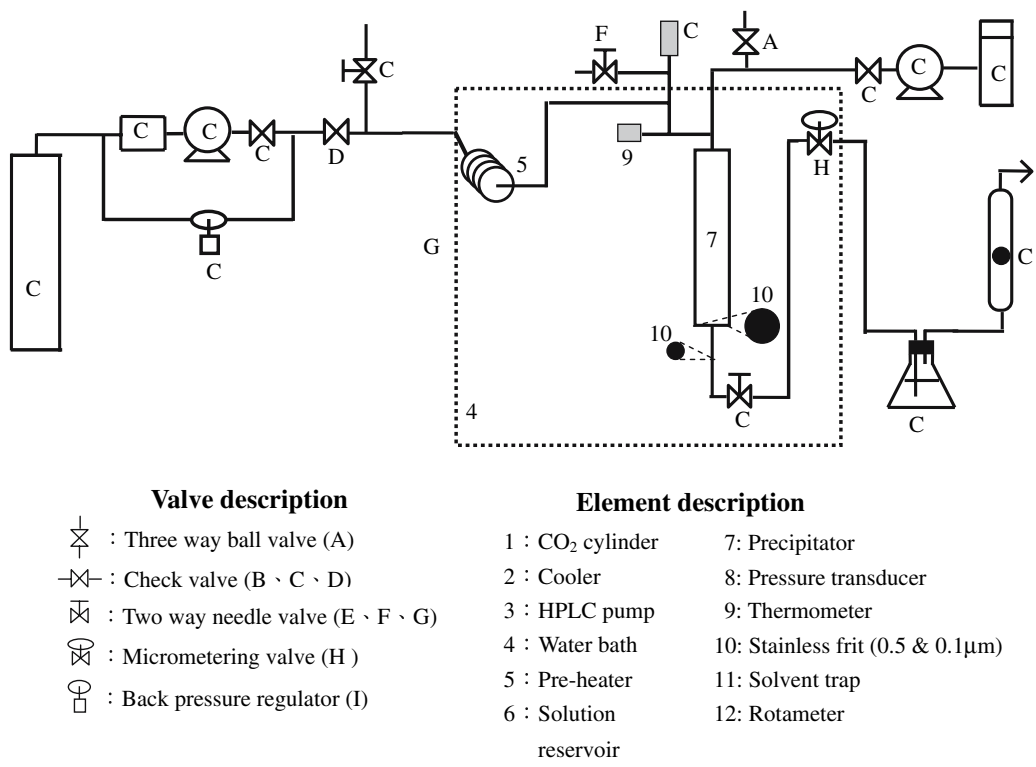


respectively. The continuous SAS experiment started by first delivering supercritical $\mathrm{CO}_{2}$ into the precipitator until the pressure and flow rate attained the steady values. The solution of sulfamethoxazole was then pumped into the precipitator through a nozzle that was a $20-\mathrm{cm}$ long stainless steel tube with an internal diameter of $127 \mu \mathrm{m}$. The solution of sulfamethoxazole contacted with the supercritical $\mathrm{CO}_{2}$ through the coaxial flow in the precipitator. Rapid recrystallization and micronization of sulfamethoxazole occurred due to the supersaturation of the solute. Similar to the batch procedures, supercritical drying process was proceeded afterwards for 1 hour to remove the residual solvent inside the precipitator. The precipitator was then depressurized and the particles precipitated on the stainless steel frits were collected for further analyses.

\section{Dissolution rate measurement}

Dissolution rates of the original and micronized sulfamethoxazole particles were performed according to the USP paddle method [7]. The experiments were conducted in a buffer solution of $400 \mathrm{~mL}$. The buffer solution had a $\mathrm{pH}$ value of 6.3 that was prepared from an aqueous solution of potassium phosphate monobasic and sodium hydroxide. The temperature of the buffer solution was kept at $37{ }^{\circ} \mathrm{C}$, and the speed of the agitator was at $100 \mathrm{rpm}$. Accurately weighed samples of pharmaceutical materials were added into the suspended basket in the buffer solution. A small amount of the buffer solution was withdrawn over a certain time interval, and the dissolved amount of sulfamethoxazole was detected using an UV spectrophotometer (Varian Cary100) at a wavelength of $261 \mathrm{~nm}$.

\section{Characterization of products}

The morphologies of sulfamethoxazole particles were examined using the scanning electron microscope (SEM, JOEL JSM-6300). Particle size distributions of sulfamethoxazole were determined using laser diffraction (LS 230, Coulter, Counter) by dispersing the powders in a $0.5 \mathrm{wt} \%$ Tween 20 aqueous solution. The crystal structures of particles were detected using the X-ray diffractometer (XRD, Philips X'pert) where data were collected between 5 and $40^{\circ}$ with a scanning rate of $5 \% \mathrm{~min}$. Thermal behavior of the particles was studied using differential scanning calorimetry (DSC, DuPont TA 2010) with a heating rate of $10 \mathrm{~K} / \mathrm{min}$.

\section{Design of experiments}

Four process variables and their influences on particle size in the continuous SAS process were evaluated using a fractional factorial design. In most literature the influences of the parameters was studied by the one-factor-at-a-time method of which the major disadvantage is the neglect of any possible interaction between factors [8]. In this study, the experimental design provided information about the main effects of the factors as well as that how these factors interacted with each other [9]. The four effects investigated included the solute concentration, solution flow rate, pressure and temperature. The Design Expert software was employed to perform the statistical analyses of the experimental data.

\section{Results and discussion}

Sulfamethoxazole was recrystallized and micronized in this study using the batch and continuous SAS processes. Comparisons of the particle sizes, morphologies, and crystal structures from both processes were presented. Sulfamethoxazole was then taken as the target pharmaceutical compound to investigate the effects of various process parameters in the continuous SAS process. The in-vitro dissolution rates of the micronized sulfamethoxazole were further measured.

Micronization of sulfamethoxazole using the SAS process

Acetone was used as the solvent in this study where the solubility of sulfamethoxazole at room temperature was determined by gravimetric method as $294.8 \mathrm{mg} / \mathrm{mL}$. The experimental temperature was $308 \mathrm{~K}$ and the solution of sulfamethoxazole was prepared at $88.4 \mathrm{mg} / \mathrm{mL}$ (30 wt\% saturation). The SEM images of the original and micronized antibiotic sulfamethoxazole is shown in Fig. 2. The untreated sulfamethoxazole showed block-like particles up to $100 \mu \mathrm{m}$ in Fig. 2a. Irregular shape particles were obtained after either the batch or continuous SAS process, as depicted in Fig. 2b and c. It is observed that the particles from the continuous SAS process indicated more significant size reduction result. Figure 3 shows the comparison of particle size distribution for sulfamethoxazole after the SAS treatments. The volume percentage distribution was adopted since it was directly correlated to the drug dosage. The mean sizes for the original sulfamethoxazole, and those from the batch and continuous SAS processes were $41.7,41.2$, and $5.1 \mu \mathrm{m}$, respectively.

The batch SAS process involved the gradual addition of an antisolvent to expand a static solution until the threshold pressure was reached and the solute precipitated. The system went across intermediate pressure conditions with incomplete mixing and nonuniform nucleation that resulted 

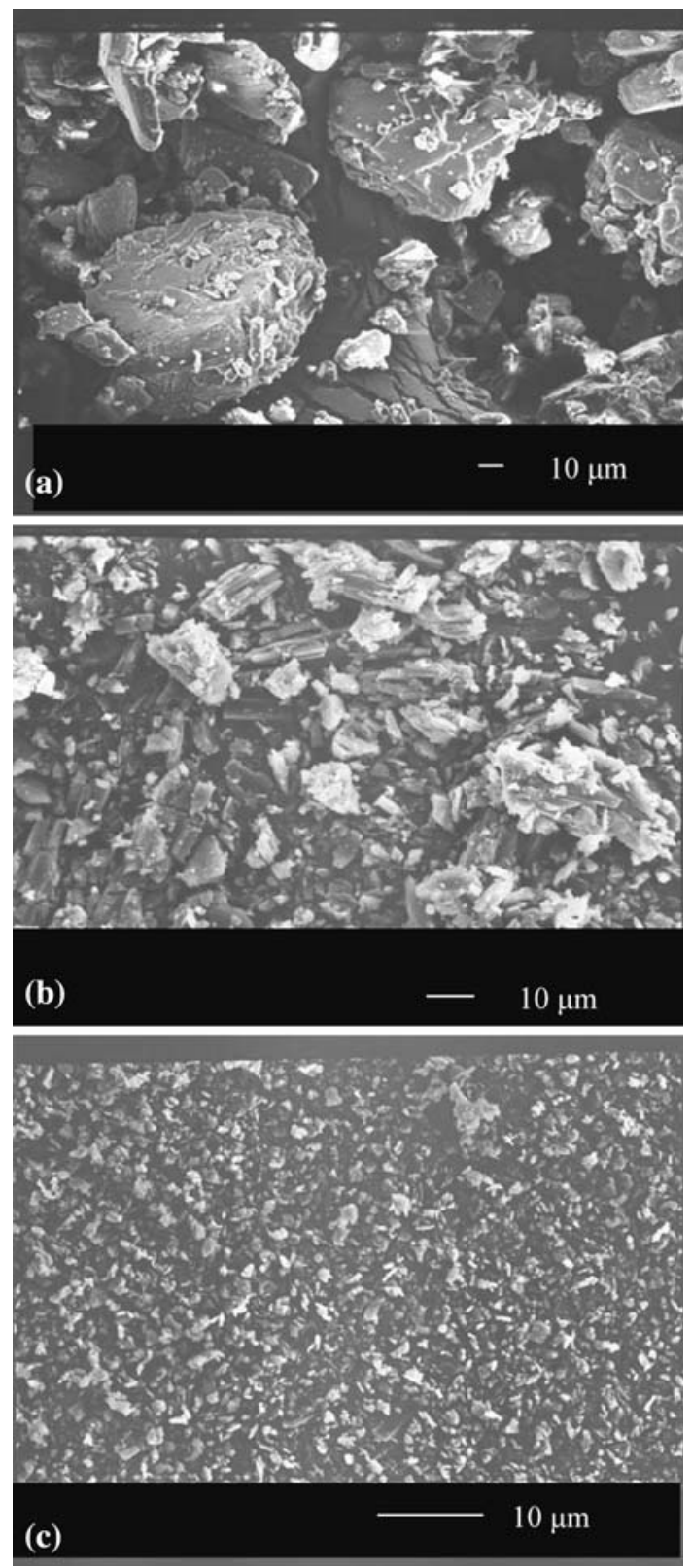

Fig. 2 SEM images of sulfamethoxazole (a) Original sulfamethoxazole (b) After the batch SAS process (308 K, 120 bar, concentration was $30 \%$ saturation (or $88.4 \mathrm{mg} / \mathrm{mL}$ ) (c) After the continuous SAS process $(308 \mathrm{~K}, 100 \mathrm{bar}$, concentration was $30 \%$ saturation (or $88.4 \mathrm{mg} / \mathrm{mL}$ ), flow rate is $0.25 \mathrm{~mL} / \mathrm{min}$ )

in relatively larger particles. The continuous SAS process involved spraying a pharmaceutical solution through a nozzle into a flowing antisolvent. The pharmaceutical solute achieved supersaturation rapidly at the constant high-pressure condition. Literature results have shown that the continuous SAS process yielded smaller particles with narrower size distribution than those from the batch mode [10]. We reported the similar experimental findings for sulfamethoxazole in this study.

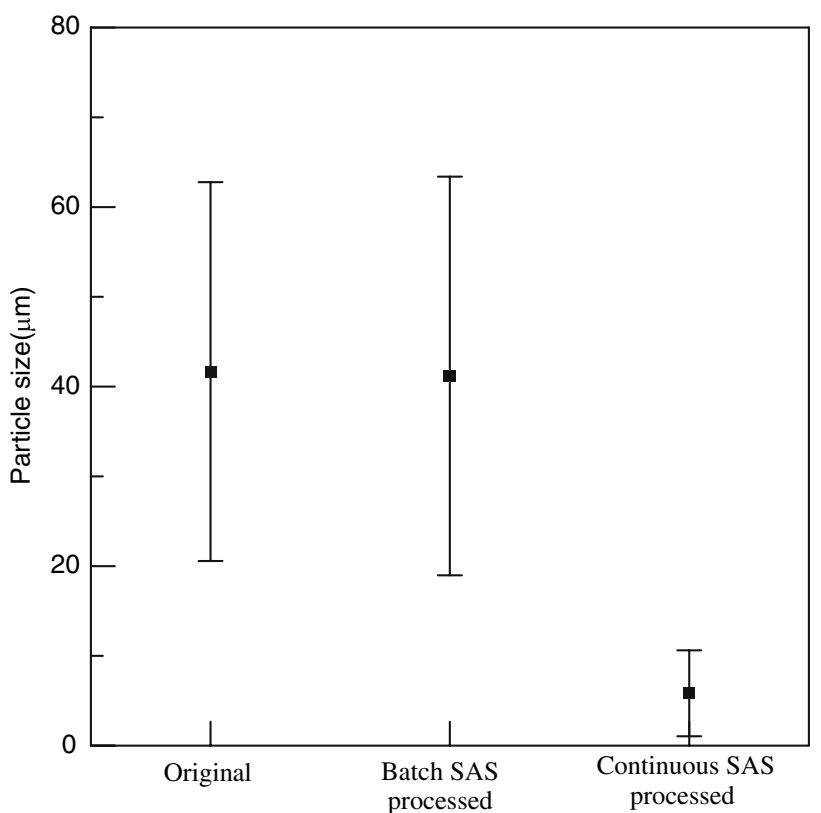

Fig. 3 Comparisons of mean particle size distribution of sulfamethoxazole

The variation of the crystal structures for sulfamethoxazole before and after the SAS precipitation was examined using XRD, and the results are presented in Fig. 4. The XRD spectra showed that the crystallinity of sulfamethoxazole was preserved after the SAS treatments, but with different intensities. The positions of characteristic peaks contributed by specific crystal planes were the same between the original and the continuous SAS treated sulfamethoxazole, as shown in Fig. 4a and c. Figure 4b, however, shows that the XRD pattern was altered after the batch SAS process. There were two new peaks at $2 \theta=16.0^{\circ}$ and $23.8^{\circ}$ that revealed a different crystal form of sulfamethoxazole was obtained. Differential scanning calorimetry (DSC) was further used to examine whether the solid-solid phase transition occurred during the SAS processes. Figure 5 shows the DSC measurement results for sulfamethoxazole. The curve from the batch SAS process shown in Fig. $5 \mathrm{~b}$ had an additional endothermic peak at $166^{\circ} \mathrm{C}$. This was confirmed as the crystal form II documented in literature [11]. The continuous SAS process produced the same crystal form $\mathbf{I}$ as the original sulfamethoxazole. It was discussed [12] that the polymorphism of drugs could be tuned by different solvents or operation conditions such as temperature and the concentration of solute. In this study, the polymorphism of sulfamethoxazole was altered by different operation modes. The particles were recrystallized throughout the precipitator in the continuous operation, while they were only collected at the bottom of the precipitator in the batch process. It was speculated that the recrystallization occurred in a 
Fig. 4 XRD results of sulfamethoxazole (a) Original sulfamethoxazole (b) After the batch SAS process (c) After the continuous SAS process
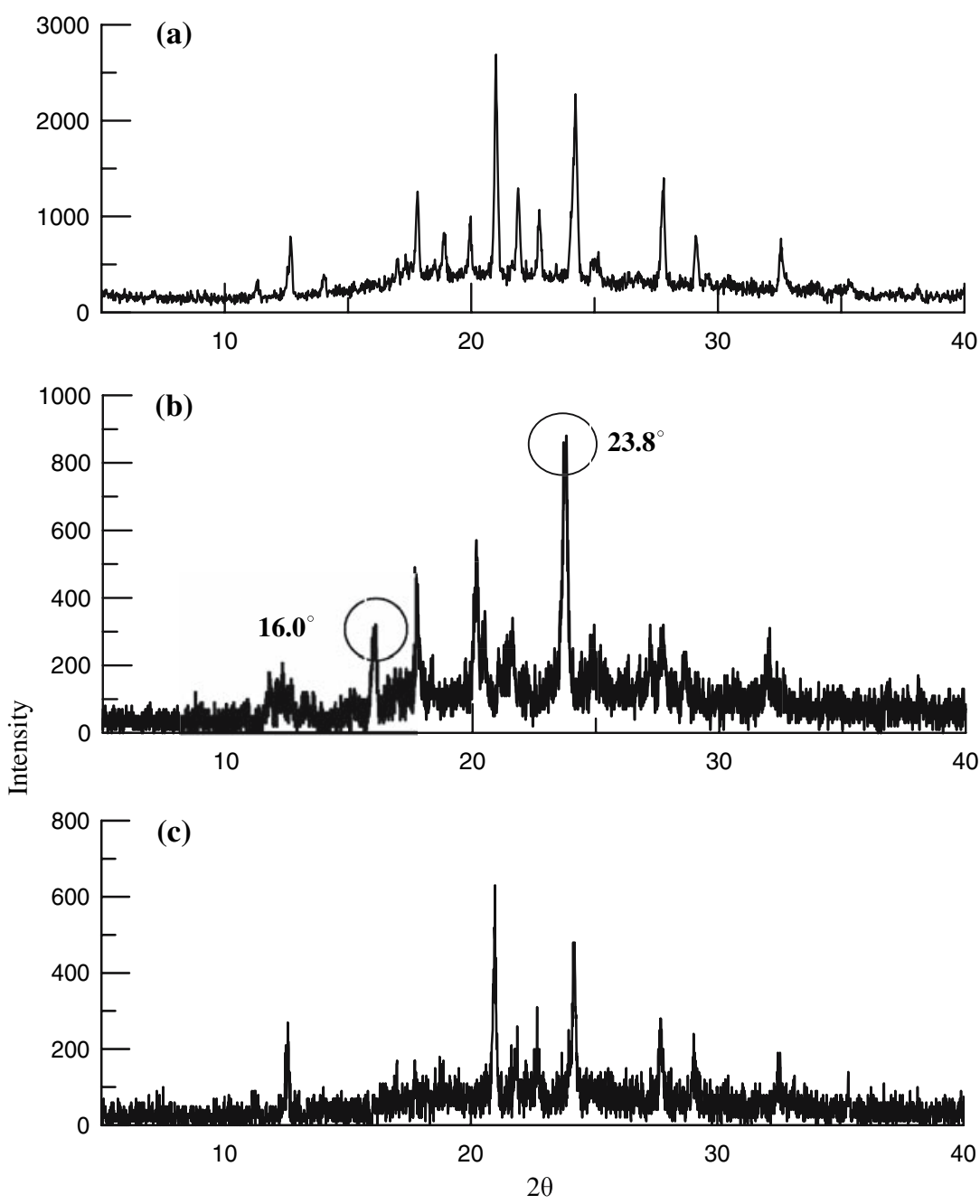

homogeneous supercritical phase during the continuous SAS operation, but in an expanded liquid phase during the batch SAS mode. Different precipitation environments in these two operation paths induced different polymorphs in the final products.

Sulfamethoxazole exhibited poor solubility in water and it had a half-life of $8-12 \mathrm{~h}$ in human body [13]. Micronization of sulfamethoxazole provided the possibility to enhance its bioavailability. The optimal operation conditions for micronizing sulfamethoxazole using the continuous process were then investigated. The dissolution rates were also tested for the feasibility of further applications.

Effects of process parameters in the continuous SAS process

In the continuous SAS process, the fractional factorial design was employed to analyze the effect of process parameters on the particle size of sulfamethoxazole. Four factors were investigated in this study: A (concentration of the pharmaceutical solution), B (solution flow rate), C (temperature), and D (pressure). Two levels for each factor were included in our experiments. Totally eight runs of the continuous SAS experiments were carried out, and the mean particle sizes with their standard deviations were summarized in Table 1. To verify the linearity assumption between the two levels of each factor, two additional center point experiments were carried out that were listed at the last two lines in Table 1. Under the level of significance $\alpha=0.05$, the $F$ distribution value was found to be $F_{0.05,1,5}=6.61$. The analysis of variance (ANOVA) was used to study the importance of each experimental factor and their cross interactions. The $F$ values of each factor were determined based on the experimental results. If the calculated $F$ value was greater than 6.61 , the factor was taken as significant on particle size. Among the factors investigated in this study, the effects of A (solution flow rate), $\mathrm{B}$ (concentration of solution), and the interaction of $\mathrm{A}$ and B were significant, as presented in Table 2. The other factors of temperature and pressure in our experimental 
Fig. 5 DSC results of sulfamethoxazole (a) Original sulfamethoxazole (b) After the batch SAS process (c) After the continuous SAS process
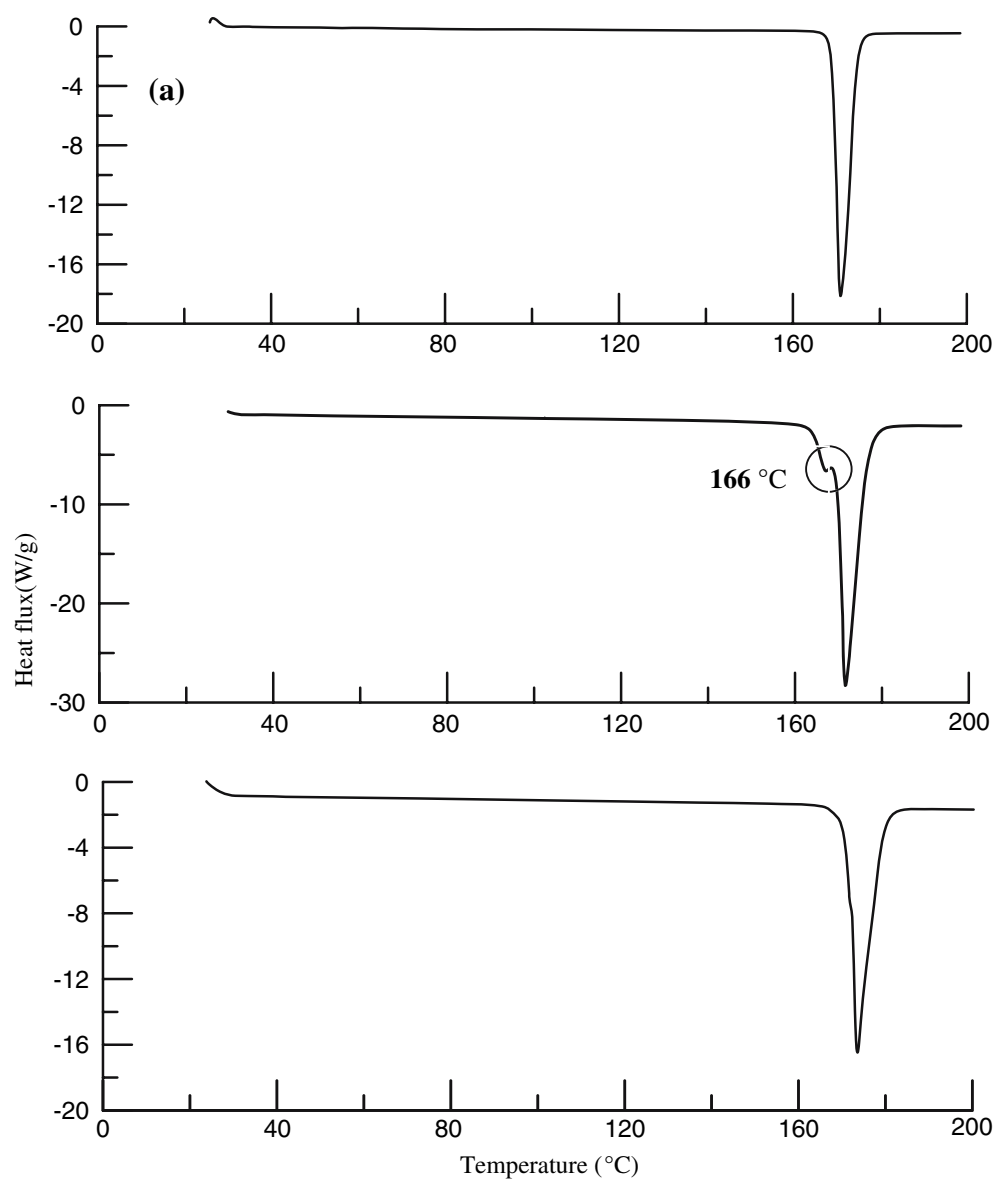

Table 1 Experimental results of the fractional factorial design

\begin{tabular}{llllrr}
\hline $\begin{array}{l}\text { Solution } \\
\text { flow rate } \\
(\mathrm{mL} / \mathrm{min})\end{array}$ & $\begin{array}{l}\text { Concentration } \\
(\% \text { sat })(\mathrm{B})\end{array}$ & $\begin{array}{l}\text { Temperature } \\
(\mathrm{K})(\mathrm{C})\end{array}$ & $\begin{array}{l}\text { Pressure } \\
(\mathrm{bar}) \\
(\mathrm{D})\end{array}$ & $\begin{array}{l}\text { Mean } \\
\text { size } \\
(\mu \mathrm{m})\end{array}$ & $\begin{array}{l}\text { Standard } \\
\text { deviation } \\
(\mu \mathrm{m})\end{array}$ \\
\hline 0.25 & 10 & 293 & 100 & 9.6 & 7.3 \\
1.00 & 10 & 293 & 120 & 13.3 & 8.3 \\
0.25 & 30 & 293 & 120 & 5.3 & 5.0 \\
1.00 & 30 & 293 & 100 & 15.2 & 14.2 \\
0.25 & 10 & 308 & 120 & 9.2 & 7.5 \\
1.00 & 10 & 308 & 100 & 12.7 & 6.5 \\
0.25 & 30 & 308 & 100 & 5.1 & 4.8 \\
1.00 & 30 & 308 & 120 & 15.4 & 14.6 \\
0.63 & 20 & 300.5 & 110 & 10.7 & 7.9 \\
0.63 & 20 & 300.5 & 110 & 10.5 & 8.2 \\
\hline
\end{tabular}

range were not significant on the effect of the particle size, and are not included in Table 2. The $F$ value for the curvature of the factors was 0.39 , thus a linear relationship in the range of two levels for factor A or B was acceptable.

The effect of the pressure on particle size was investigated at 100 and 120 bar in this study. The critical pressure for the binary mixture of $\mathrm{CO}_{2}$ and acetone was 72 bar at
Table 2 Result from the ANOVA for the mean particle size

\begin{tabular}{llccr}
\hline $\begin{array}{l}\text { Source of } \\
\text { variation }\end{array}$ & $\begin{array}{l}\text { Degree of } \\
\text { freedom }\end{array}$ & $\begin{array}{l}\text { Sum of } \\
\text { squares }\end{array}$ & $\begin{array}{l}\text { Mean } \\
\text { square }\end{array}$ & $F$-value \\
\hline A & 1 & 93.8 & 93.8 & 1466.3 \\
B & 1 & 1.8 & 1.8 & 28.2 \\
AB & 1 & 21.1 & 21.1 & 330.1 \\
Curvature & 1 & 0.025 & 0.025 & 0.39 \\
\hline
\end{tabular}

308.15 K [14]. Although the critical pressure would increase as sulfamethoxazole was added into this binary solution $[15,16]$, the operation pressures in this study were well above the two-phase region. This explains that pressure had little effect on particle size over our pressure range.

Temperature was a competing effect in the SAS process. The degree of supersaturation of the sulfamethoxazole solute decreased with increasing temperature. This is not favorable for small particle formation. On the other hand, the nucleation rate of solute increased with increasing temperature, and resulted in a favorable condition for small particle formation. Similar to other literature results [17, 18], temperature had negligible effect for the recrystallization of sulfamethoxazole in this study. 
The results of ANOVA showed that an interaction effect existed between the concentration and flow rate of the sulfamethoxazole solution. At a lower flow rate ( $0.25 \mathrm{~mL} / \mathrm{min})$, a higher concentration (30\% saturation) resulted in smaller particles whereas the opposite tendency was observed at a higher solution flow rate $(1.0 \mathrm{~mL} / \mathrm{min})$. In a continuous SAS process the solution flow rate could affect the hydrodynamics in the precipitator. At a lower flow rate, the relative velocity between the solution and $\mathrm{CO}_{2}$ favored macromixing. The pharmaceutical solute nucleated at a uniform supersaturation status, and enabled little opportunity for crystal growth. With a higher concentration at this lower flow rate, a higher nucleation rate resulted in smaller particle formation. At a high solution flow rate, there might exist an incomplete mixing and a gradient of supersaturation. This resulted in an enhanced crystal growth and the formation of larger particles. Figure 6 shows the comparison for the particle morphologies of sulfamethoxazole at different solution flow rates. It is demonstrated that the smaller and irregular particles were obtained at a lower solution flow rate, as shown in Fig. 6a. The rod-like and larger particles shown in Fig. $6 \mathrm{~b}$ indicated that the particle
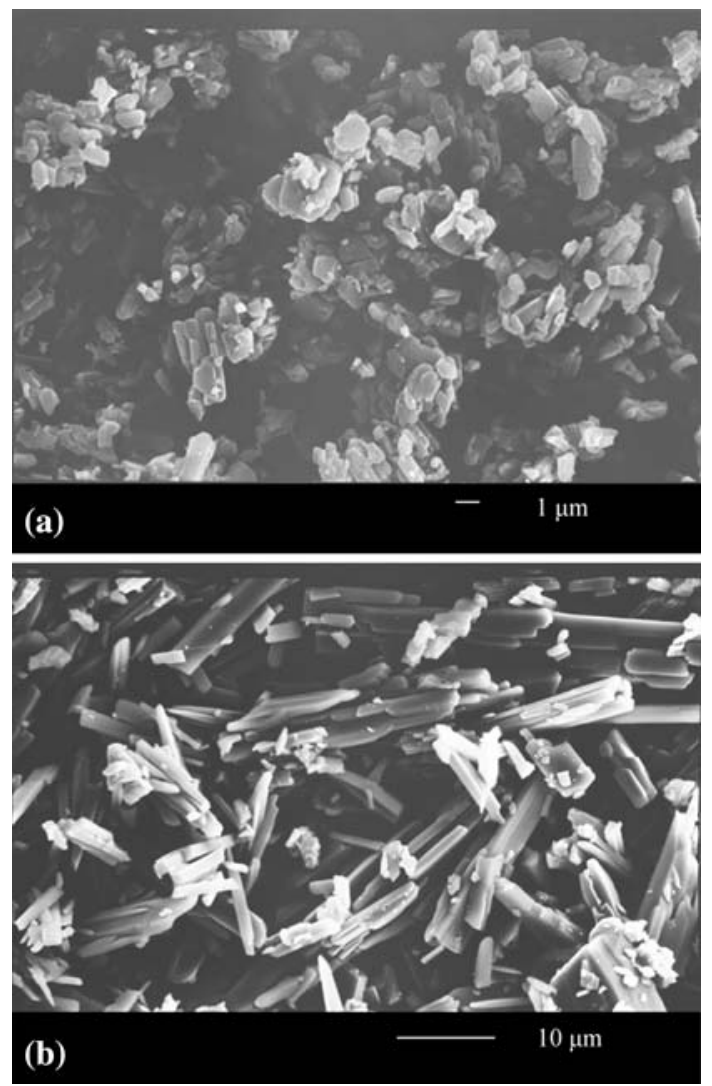

Fig. 6 SEM image of sulfamethoxazole under different flow rates (a) $0.25 \mathrm{~mL} / \mathrm{min}$ (308 K, $100 \mathrm{bar}$, concentration was $30 \%$ saturation (or $88.4 \mathrm{mg} / \mathrm{mL}))($ b) $1.00 \mathrm{~mL} / \mathrm{min}(308 \mathrm{~K}, 120 \mathrm{bar}$, concentration was $30 \%$ saturation (or $88.4 \mathrm{mg} / \mathrm{mL}$ )) formation is growth rate controlled. The interaction effect between the concentration and the solution flow rate was also discussed in previous literature [19, 20].

From the above discussions, the optimal operation conditions for the micronization of sulfamethoxazole using the continuous SAS process were $100 \mathrm{bar}, 308 \mathrm{~K}$, and a flow rate at $0.25 \mathrm{~mL} / \mathrm{min}$ with a concentration at $30 \mathrm{wt} \%$ of saturation (or $88.4 \mathrm{mg} / \mathrm{mL}$ ) in acetone.

Results for the dissolution rate of sulfamethoxazole

The micronized sulfamethoxazole obtained at the optimal operation conditions was further tested for its dissolution rate in a simulated intestinal fluid. The dissolution profiles for the original and micronized sulfamethoxazole are shown in Fig. 7. It is depicted that $95 \%$ of the micronized sulfamethoxazole was dissolved after $10 \mathrm{~min}$. This dissolution rate was three times faster than the original sulfamethoxazole, and was attributed to the reduction in its mean particle size from 41.7 to $5.1 \mu \mathrm{m}$.

Additives like polymers or surfactants were often incorporated in drug formulation which was another approach to enhance the solubility or dissolution rate of drugs besides the micronization technique [21]. In this study, a co-precipitation experiment was carried out using a hydrophilic polymer hydroxypropylcellulose (HPC). Sulfamethoxazole was micronized using a continuous SAS process at the optimal conditions, and HPC was also added in the solution at $10 \mathrm{wt} \%$ relative to the amount of

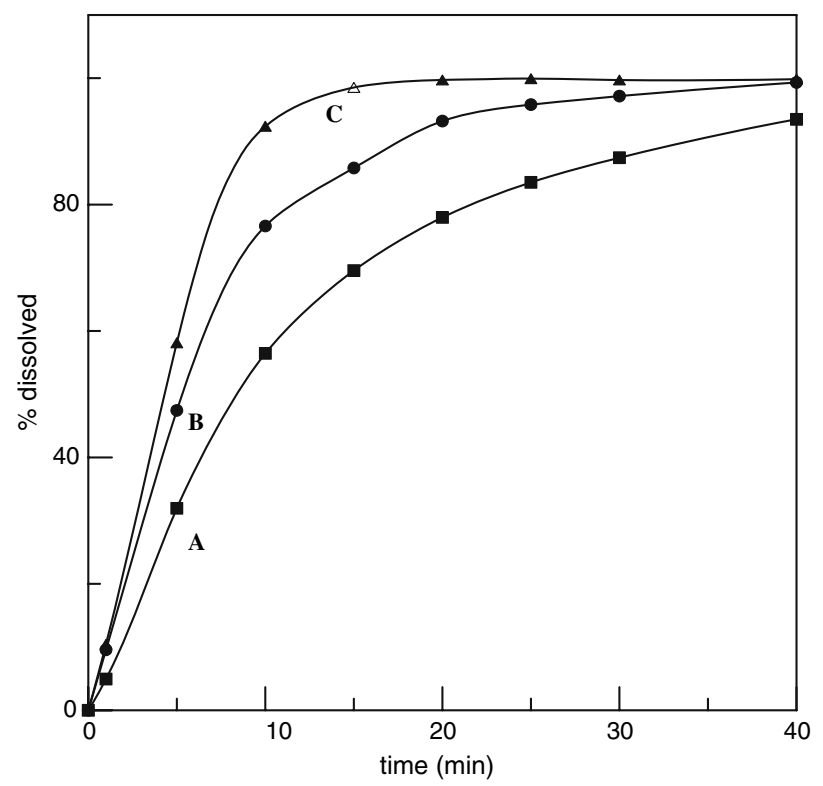

Fig. 7 Dissolution profile of sulfamethoxazole (A) the original sulfamethoxazole (B) the micronized sulfamethoxazole from the continuous SAS process (C) the sulfamethoxazole with the co-precipitated HPC 
sulfamethoxazole in acetone. The product of the co-precipitation process was aggregates of sulfamethoxazole and HPC. The dissolution rate of the aggregates was also shown in Fig. 7. It was observed that $95 \%$ of sulfamethoxazole was dissolved after one min. This much faster dissolution rate was owing to the hydrophilic nature of HPC.

\section{Conclusion}

Micronized particles of sulfamethoxazole were satisfactorily precipitated from acetone using the SAS processes. Two modes of the batch and continuous SAS process were compared and the latter yielded smaller particles. The optimal operation conditions for sulfamethoxazole were investigated using the fractional factorial design method. At the optimal conditions (100 bar, $308 \mathrm{~K}$, flow rate of $0.25 \mathrm{~mL} / \mathrm{min}$, and solute concentration of $88.4 \mathrm{mg} / \mathrm{mL}$ ), the mean particle size of sulfamethoxazole was micronized from its original 41.7 to $5.1 \mu \mathrm{m}$. The dissolution rate of the micronized sulfamethoxazole in a simulated intestinal fluid was significantly enhanced. The co-precipitation of sulfamethoxazole with a hydrophilic polymer HPC was also investigated. The dissolution rate of the co-precipitated aggregates showed a much faster result due to the hydrophilic nature of the additive.

Acknowledgements The authors are grateful to the support of the Ministry of Economic Affairs, ROC, for supporting this study under the project of Green Process Technology (94-EC-17-A-09-S1-019).

\section{References}

1. Pasquali I, Bettini R, Giordano F (2006) Eur J Pharm Sci 27:299_ 310

2. Bahrami M, Ranjbarian S (2007) J Supecrit Fluids 40:263-283

3. Shargel L, Yu A (1999) Applied biopharmaceutics and pharmacokinetics, 4th edn. McGraw-Hill, New York

4. Shekunov B, York P (2000) J Crystal Growth 211:122-136

5. Reverchon E (1999) J Supercrit Fluids 15:1-21

6. Su CS, Chen YP (2005) Chem Eng Technol 28:1177-1181

7. United States Pharmacopedia Convention Inc. (2005) The United States pharmacopedia section: test solutions, 28th edn. United States Pharmacopedia Convention Inc., Washington

8. Subra P, Jestin P (2000) Ind Eng Chem Res 39:4178-4184

9. Douglas C (1997) Design and analysis of experiments, 3rd edn. Wiley, New York

10. Warwick B, Dehghani F, Foster N, Biffin J, Regtop H (2002) Ind Eng Chem Res 41:1993-2004

11. Maury L, Rambaud J, Pauvert B, Lasserre Y, Audran B (1985) Can J Chem 63:3012-3018

12. Edwards A, Shekunov B, Kordikowski A, Forbes R, York P (2001) J Pharm Sci 90:1115-1124

13. Katzung B (2004) Basic and clinical pharmacology, 9th edn. McGraw-Hill, New York

14. Day CY, Chang CJ, Chen CY (1996) J Chem Eng Data 41:839843

15. Reverchon E, Caputo G, De Marco I (2003) Ind Eng Chem Res 41:6406-6414

16. Reverchon E, De Marco I (2004) J Supercrit Fluids 31:207-215

17. Krober H, Teipel U (2002) J Supercrit Fluids 22:229-235

18. Wubbolts F, Bruinsma O, van Rosmalen, GM (1999) J Crystal Growth 198/199:767-772

19. Haleblian J (1975) J Pharm Sci 64:1269-1288

20. Magnan C, Badens E, Commenges N, Charbit G (2000) J Supercrit Fluids 19:69-77

21. Leuner C, Dressman J (2000) Eur J Pharm Biopharm 50:47-60 